\title{
Performance of Two Screening Questionnaires for Inflammatory Arthritis in Patients with Inflammatory Bowel Disease
}

\author{
Rubén Queiro (D), ${ }^{1}$ Sergio Rodríguez-Caminero, ${ }^{1}$ Sabino Riestra, ${ }^{2}$ Ruth de Francisco, ${ }^{2}$ \\ Isabel Pérez-Martínez $\mathbb{D}^{2}{ }^{2}$ and Javier Ballina ${ }^{1}$ \\ ${ }^{1}$ Rheumatology Division, Hospital Universitario Central de Asturias (HUCA), Oviedo, Spain \\ ${ }^{2}$ Gastroenterology Division, IBD Unit, Hospital Universitario Central de Asturias (HUCA), Oviedo, Spain
}

Correspondence should be addressed to Rubén Queiro; rubenque7@yahoo.es

Received 20 February 2018; Revised 22 March 2018; Accepted 1 April 2018; Published 8 May 2018

Academic Editor: David Bernardo

Copyright (c) 2018 Rubén Queiro et al. This is an open access article distributed under the Creative Commons Attribution License, which permits unrestricted use, distribution, and reproduction in any medium, provided the original work is properly cited.

\begin{abstract}
Musculoskeletal symptoms are the most common extraintestinal manifestations of inflammatory bowel disease (IBD). An essential step in the management of these patients is to establish referral algorithms through the use of appropriate screening tools. Our objective was to evaluate the performance of two simple questionnaires to detect inflammatory arthritis (IA) in patients with IBD. Two questionnaires, one for detecting axial IA and the other for peripheral IA, were tested among 112 IBD unselected consecutive patients of both sexes, aged 18-45 years. The study period was from January to December 2016. Each questionnaire was composed of three simple questions. If the patient answered affirmatively at least to two of the three questions, the questionnaire was considered positive. Clinical diagnosis of IA based on an expert's opinion was the reference gold standard. To obtain a weighted value of sensitivity and specificity, likelihood ratio (LR) values were calculated. Twenty-seven percent of the patients were considered positive responders to the axial questionnaire while $32 \%$ were considered positive responders to the peripheral questionnaire. Twenty-four patients (21.4\%) were diagnosed with axial IA, whereas $26 \%$ had peripheral IA. The axial questionnaire yielded a sensitivity of $87.5 \%$ (67.6-97.3), specificity of $89.8 \%(81.5-92.2)$ and LR+ of 8.6 (4.5-16.2). For the peripheral questionnaire, these values were $82.8 \%$ (64.2-94.2), 87.4\% (79-93.3), and 6.6 (3.8-11.4), respectively. Both questionnaires showed an adequate screening capacity for IA in patients with IBD. Their specificity, together with their simplicity, can make them suitable detection tools in gastroenterology and general medicine consultations.
\end{abstract}

\section{Introduction}

Inflammatory bowel disease (IBD) is a chronic immunemediated disease that affects the gastrointestinal tract. It is comprised of two subtypes: Crohn's disease (CD) and ulcerative colitis (UC). Both are thought to result from complex stochastic interactions between aberrant immune responses to gut luminal microbes in genetically susceptible individuals who are exposed to environmental risk factors [1-3]. IBD is most common in North America and western and northern Europe, where incidence rates of $\mathrm{UC}$ and $\mathrm{CD}$ range from 2.2 to 24.3 per $10^{5}$ person-years [3].

Musculoskeletal symptoms (MSs) are the most common extraintestinal manifestations in patients with IBD. Many of these IBD-associated MSs belong to the broader concept of spondyloarthritis (SpA) [4-7]. The prevalence of inflammatory rheumatic manifestations is highly variable in IBD. In a recent meta-analysis, the pooled prevalence rates of rheumatic diagnoses were as follows: sacroiliitis, 10\%; ankylosing spondylitis, $3 \%$; peripheral arthritis, $13 \%$; enthesitis, $1 \%$ to $54 \%$; and dactylitis, $0 \%$ to $6 \%$ [8]. However, when all these manifestations of the SpA spectrum are grouped together, more than one-third of IBD patients show the SpA features included in the new Assessment of SpondyloArthritis International Society (ASAS) criteria $[9,10]$.

Some IBD patients may show peripheral/axial arthritis that sometimes runs a parallel course with gut inflammation, while in others, joint and gut inflammation run independent courses $[4-7,11]$. In daily practice, symptoms of SpA, either axial or peripheral, are not adequately recognized by either 
patients or gastroenterologists [9]. To most patients, the relationship between joint and gut symptoms is unknown, and gastroenterologists do not always specifically ask patients about joint involvement. Consequently, patients with symptoms of SpA may be underdiagnosed and effective treatment may be delayed. In a study that included 350 unselected patients with IBD, half of the patients who reported at least one musculoskeletal SpA feature never visited a rheumatologist. Axial or peripheral SpA was diagnosed in 58\% of the patients who were examined by a rheumatologist, and in $21 \%$, another rheumatic disorder was diagnosed [9].

Therefore, the collaboration between rheumatologists and gastroenterologists should be strengthened, as both gut and joint inflammations can seriously deteriorate the quality of life of patients. In many cases, the therapeutic decision process is common to both conditions $[12,13]$. A key part of this collaboration is to establish appropriate derivation algorithms from one specialty to another, without these algorithms implying an excessive assistance load for any of the specialties involved $[12,13]$. Ideally, these tools should be valid and easy to use in routine clinical practice.

We aimed to evaluate the diagnostic performance of two screening questionnaires for inflammatory arthritis (IA) in subjects with IBD. The simplicity of these questionnaires could lead to improved care for patients with IBD and suspected SpA.

\section{Patients and Methods}

The participants were prospectively recruited from a single university institution in northern Spain. The inclusion criteria were patients of both sexes aged 18-45 years, a verified diagnosis of IBD (based on endoscopic, laboratory, and histological findings), and ability to provide written informed consent. The exclusion criteria were patients with a known rheumatologic or musculoskeletal condition, patients involved in a labor litigation, patients awaiting recognition of incapacity, or those with temporary or permanent disabilities due to their IBD, and those found to be unable to comply with the study procedures. The inclusion period was from January 2016 to December 2016.

All the patients provided their informed written consent. In accordance with the Spanish recommendations, the study was approved by the Clinical Research Ethics Committee of Hospital Universitario Central de Asturias (reference number HUCA 67/14) and was conducted in accordance with the principles of the Declaration of Helsinki for studies on humans.

This study was developed in several steps. First, two rheumatologists with expertise in SpA agreed on three questions for the detection of axial arthritis and three questions for the detection of peripheral arthritis.

The three questions agreed upon for peripheral arthritis were as follows:

(i) Do you have joint pain?

(ii) When waking up in the morning, do you notice stiffness in your joints for a time equal to or greater than 30 minutes?

\section{(iii) Do you have or have you had any swollen joint?}

The overall questionnaire response was considered positive if the patient answered affirmatively to at least two of the three questions.

The three questions agreed upn for axial arthritis were as follows:

(i) Do you have back pain?

(ii) When waking up in the morning, do you notice stiffness in your back for a time equal to or greater than 30 minutes?

(iii) Do you have or have you had back pain that wakes you up or interrupts your sleep?

The overall questionnaire response was considered positive if the patient answered affirmatively to at least two of the three questions.

To avoid including patients with degenerative joint diseases that could bias the positivity of the questionnaire responses, only adult patients aged $\leq 45$ years were included. This age limit is the same as that used in the ASAS criteria for SpA [10].

The clinical part of the study consisted of detailed clinical history, family medical history, physical examination, and laboratory and imaging tests (except ultrasonography (US) and according to the criteria of the evaluating physician). The final diagnosis of IA, either axial or peripheral, was established by a rheumatologist with extensive experience in SpA.

Second, all the patients underwent a US study of peripheral joints and entheses. This part of the study was conducted by an expert in musculoskeletal US, who was blinded to the clinical part of the study. The US examination was performed with MyLab 70XVG (Esaote S.p.A., Genova, Italy). Details of this US protocol have been published elsewhere $[14,15]$. Enthesitis, synovitis, synovial effusion, synovial hypertrophy, positive power Doppler (PD) signal, and so forth were defined in accordance with the standards provided by OMERACT's musculoskeletal US working group [16].

2.1. Statistical Methods. A descriptive statistical analysis of all the variables was performed, including central tendency and dispersion measures for continuous variables and absolute and relative frequencies for categorical variables. Student's $t$ test, Mann-Whitney $U$ test, or Kruskal-Wallis $H$ test was used to compare quantitative variables, and Pearson's chi-square or Fisher's exact test was used for qualitative variables. Tests were two-tailed with a significance level of 5\%. Data were analyzed using the SPSS v19.0 statistical software.

The sample size was calculated to achieve an accuracy of $10 \%$ using a normal bilateral asymptotic $95 \%$ confidence interval (CI), assuming a sensitivity of $80 \%$. To evaluate sensitivity and specificity, both questionnaires were tested in the whole study population and among 110 healthy subjects. The healthy control population was matched by age $(34 \pm$ $6.8 \mathrm{yrs}$ ) and sex (44.5\% females and $55.5 \%$ males) with the study population. Subjects with a personal history of inflammatory rheumatic diseases or a family history of psoriasis or inflammatory rheumatic processes were excluded as controls. 


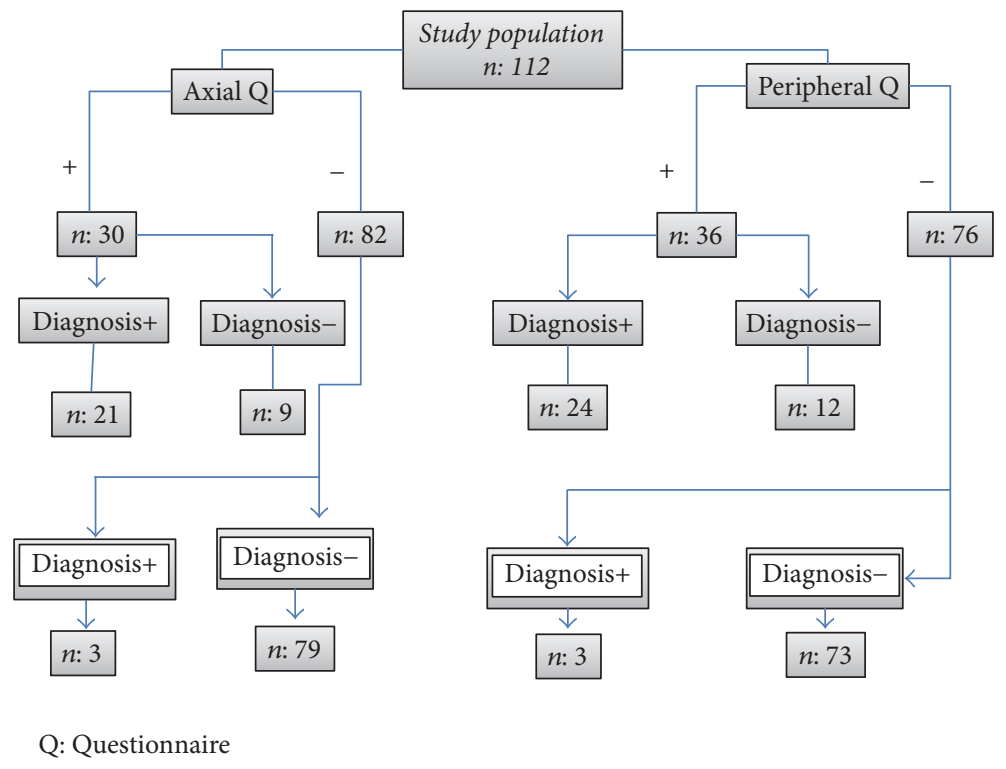

FIgURE 1: Flow chart according to the answers to the questionnaires.

Test-retest reliability, sensitivity, specificity, positive/negative $L R$, and positive/negative predictive values were calculated for both questionnaires. Intrarater reliability (Cohen's kappa) was calculated for the US studies conducted in the study population and in 30 healthy age- and sex-matched controls.

\section{Results}

Of the 112 patients, 48 (42.9\%) were women and 64 (57.1\%) were men, with a mean age of $33 \pm 7.2$ years. Fortyfour cases were UC, and 68 were $\mathrm{CD}$. The mean duration of IBD was $8 \pm 5.6$ years. As for treatments, 42 patients received biological therapy, and 51 received classical immunosuppressants (mostly thiopurines). We found no significant differences with respect to age, sex, duration of illness, level of education, BMI, or IBD family history between CD and UC. Table 1 represents the main disease characteristics of the study population.

Based on the questionnaire responses, $27 \%$ of the patients were considered positive responders to the axial questionnaire and $32 \%$ were considered positive responders to the peripheral questionnaire. Twenty-four patients (21.4\%) were diagnosed with axial IA, whereas $26 \%$ had peripheral IA. Sensitivity, specificity, and LR+ values for the axial questionnaire were $87.5 \%, 89.8 \%$, and 8.6 , respectively, whereas for the peripheral questionnaire, these values were $82.8 \%, 87.4 \%$, and 6.6, respectively. In the control population, specificity for the axial and peripheral questionnaires was $90.7 \%$ (83.6-95.5) and $89.6 \%$ (82.4-94.7), respectively. The test-retest reliability revealed an excellent CCI of $0.94(0.92-0.99)$ for both questionnaires. Figure 1 represents the flow chart of the study population according to questionnaire responses.

These results were not affected by exposure to systemic treatments. The LR+ of the axial arthritis questionnaire was $7.6(3.8-16.7)$ in the patients not exposed to systemic therapies
TABLE 1: Disease characteristics of the study population.

\begin{tabular}{lc}
\hline Disease feature & Study population $N: 112$ \\
\hline Age $(\mathrm{yrs}$, mean $\pm \mathrm{SD})$ & $33.12 \pm 7.19$ \\
Disease duration (yrs, mean $\pm \mathrm{SD})$ & $8 \pm 5.6$ \\
BMI $\left(\mathrm{Kg} / \mathrm{m}^{2}\right)$ & $23.3 \pm 4.5$ \\
Women & $48(42.9 \%)$ \\
Men & $64(57.1 \%)$ \\
UC & $44(39.3 \%)$ \\
CD & $68(60.7 \%)$ \\
IBD family history & $21(18.7 \%)$ \\
Biologics & $42(37.5 \%)$ \\
Classic immunosuppressants & $51(45.5 \%)$ \\
Corticoids & $11(9.8 \%)$ \\
IBD surgery & $28(25 \%)$ \\
Diabetes & $0(0 \%)$ \\
Hypertension & $0(0 \%)$ \\
Dyslipidemia & $2(1.8 \%)$ \\
Smokers & $24(21.4 \%)$ \\
Alcohol & $25(22.3 \%)$ \\
Psoriasis & $11(9.8 \%)$ \\
Psoriasis family history & $14(12.5 \%)$ \\
Asthma & $14(12.5 \%)$ \\
Clinical enthesitis* & $5(4.5 \%)$ \\
Dactylitis & $3(2.7 \%)$ \\
\hline
\end{tabular}

BMI: body mass index; UC: ulcerative colitis; CD: Crohn's disease; IBD: inflammatory boweldisease. ${ }^{*}$ After clinical examination.

and 8.4 (4.4-15.6) in those exposed to these drugs. For the peripheral arthritis questionnaire, the LR+ value was 6.7 (3.8-13.8) in patients not exposed to systemic therapies and $7.4(4.3-12.8)$ in those exposed to systemic therapies. 
TABLE 2: Reliability parameters of the screening questionnaires.

\begin{tabular}{lccc}
\hline Parameter & Axial Q versus clinical D & Per. Q versus clinical D & US versus clinical D \\
\hline Sensitivity & $87.5 \%(67.6-97.3)$ & $82.8 \%(64.2-94.2)$ & $50.5 \%(36.4-71.9)$ \\
Specificity & $89.8 \%(81.5-92.2)$ & $87.4 \%(79-93.3)$ & $64.9(53.2-75.4)$ \\
Positive LR & $8.6(4.5-16.2)$ & $6.6(3.8-11.4)$ & $1.6(1.1-2.4)$ \\
Negative LR & $0.14(0.05-0.4)$ & $0.2(0.09-0.4)$ & $0.7(0.5-1)$ \\
PPV & $70 \%(50.6-85.3)$ & $66.7 \%(49-81.4)$ & $40 \%(25.7-55.7)$ \\
NPV & $96.3 \%(89.7-99.2)$ & $94.3 \%(87.2-98.1)$ & $76.9 \%(64.8-88.5)$ \\
\hline
\end{tabular}

LR: likelihood ratio; Q: questionnaire; Per: peripheral; D: diagnosis; US: ultrasound; PPV: positive predictive value; NPV: negative predictive value.

Intrarater reliability was excellent in both US substudies (0.93 [0.92-0.98]). More US changes were found in the study population with respect to the control group. The percentage of patients with synovial effusion and/or synovial hypertrophy was greater in the study population than in the control group ( $31 \%$ versus $10 \%, p=0.001)$. More structural changes were detected in the patients' entheses than in those of the controls $(41.9 \%$ versus $13.3 \%, p=$ $0.0001)$. More patients (40.2\%) had findings of enthesitis and/or active synovitis (PD positive signal) than the controls (3.3\%; $p<0.0001)$. The sensitivity, specificity, and LR+ of US examination for clinical diagnosis of IA were $50.5 \%, 64.9 \%$, and 1.6 , respectively $(p=0.046)$.

We found no statistically significant associations between the positive questionnaire responses and the positive US findings. However, when the responses to both questionnaires were negative, the US findings were also negative, with a specificity of $89.2 \%$ (79.1-95.6). Table 2 summarizes the reliability of the questionnaire responses and US findings.

\section{Discussion}

In this study, we verified that two simple questionnaires were consistent with regard to the detection of IA in patients with IBD, with high sensitivity and specificity. On the other hand, in this unselected population, the prevalence of IA with features of SpA was high. Almost one-fourth of the study population had IA according to the criteria of a rheumatologist expert in SpA. These data are in line with prevalence data from earlier studies, thus reinforcing the reliability of both questionnaires [4-7].

The two questionnaires tested here showed a high sensitivity and specificity, so their predictive capacity for IA may be useful in daily practice for gastroenterologists and family medicine doctors, thus leading to improved referrals for rheumatology consultations. Such ability depends largely on the pretest probability of finding a true case of IBDassociated arthritis. Following the principles of the Bayes theorem, if the expected prevalence of IA in this population is intermediate-high (30-50\%) according to the international literature, for a positive LR of approximately 6-9, which were the values obtained from our study, we would obtain posttest probabilities of arthritis of 70-80\%. In other words, of 10 patients with IBD who might answer positively to either questionnaire, between 7 and 8 could actually have IBD-associated arthritis. In addition, if we take into account the fact that both questionnaires are simple and easy to implement in everyday practice, it is worthwhile to test their usefulness in other centers to obtain an external validation of these results. Together, both questionnaires yielded a high specificity $(\approx 90 \%)$, which indicates that they have the potential to determine which patients should (or not) be sent to rheumatology appointments.

We found a high prevalence of US findings both in the joints and in the entheses of these patients. These findings were significantly higher than those found in the healthy control population matched by age and sex. On the other hand, the association between our US findings and the clinical questionnaire responses was weak or nonexistent. In other studies of musculoskeletal US performed in subjects with IBD, a high prevalence of subclinical US abnormalities has been found $[17,18]$. Something similar has been published in patients with psoriasis without arthritis [19]. Therefore, our US data are in line with those of other studies in IBD or psoriasis and reflect the subclinical nature of most of them. The clinical significance of these findings will need to be elucidated in prospective studies with enough long follow-up. Moreover, the prevalence of these findings was not affected by exposure to systemic therapies (data not shown). When the responses to both questionnaires were negative, no positive findings were found on US (specificity close to 90\%). This reinforces, once again, the high reliability of both questionnaires.

Questionnaires showed adequate sensitivity and specificity both in exposed patients and in those not exposed to systemic treatments. This is an unexpected finding. We could not make an exact correction according to the past or current exposure to systemic therapies. When stratifying patients according to their exposure to this type of treatment, comparisons were reduced to relatively small groups of patients, and this could have been associated with a decrease in the statistical power of the study (type II error). Therefore, this finding should be taken with caution.

Some weaknesses of the study should be highlighted. One is that the study was conducted in a single university center that generally addresses the most serious cases of IBD. Therefore, we do not know if these questionnaires would work equally well in other clinical settings. The clinical diagnosis of IA was based on the judgment of a physician expert in SpA, but it was not contrasted to other specialists' diagnoses. It also was not clearly tested against the ASAS criteria for axial or peripheral SpA [10]. Therefore, we did not generate information about its consistency. In any case, the ASAS criteria are for classification purposes and cannot replace the diagnosis made by an SpA expert [10, 20]. Having 
chosen a young population (age limit of 45 years), we do not know how these instruments would behave in populations of patients with IBD who are above 45 years of age. Finally, the questionnaires were taken as a whole, without item-by-item weighting.

Within the strengths of this study, the simplicity and comfort of the questionnaires for physicians and patients are worth mentioning. In fact, the test-retest reliability (performed with an average interval of 2 weeks) was very high for both. The fact that almost one-fourth of the patients in this series had IA (a figure very similar to that reported in other studies) can be assumed as adequate face and content validities for both questionnaires.

In summary, we tested the usefulness of two simple questionnaires for detecting arthritis in patients with IBD. Both were better than US for the aforementioned purpose. Validation of the results of this study in other clinical settings would be interesting.

\section{Data Availability}

All the clinical data of this study are conserved in databases of the gastroenterology and rheumatology services of HUCA, Avda. de Roma s/n, 33011 Oviedo, Spain.

\section{Conflicts of Interest}

The authors declare no conflicts of interest regarding the publication of this paper.

\section{References}

[1] D. H. Kim and J. H. Cheon, "Pathogenesis of inflammatory bowel disease and recent advances in biologic therapies," Immune Network, vol. 17, no. 1, pp. 25-40, 2017.

[2] K. J. Maloy and F. Powrie, "Intestinal homeostasis and its breakdown in inflammatory bowel disease," Nature, vol. 474, no. 7351, pp. 298-306, 2011.

[3] A. N. Ananthakrishnan, "Epidemiology and risk factors for IBD," Nature Reviews Gastroenterology \& Hepatology, vol. 12, no. 4, pp. 205-217, 2015.

[4] S. Ditisheim, N. Fournier, P. Juillerat et al., "Inflammatory articular disease in patients with inflammatory bowel disease: result of the Swiss IBD cohort study," Inflammatory Bowel Diseases, vol. 21, no. 11, pp. 2598-2604, 2015.

[5] J. D. Olpin, B. P. Sjoberg, S. E. Stilwill, L. E. Jensen, M. Rezvani, and A. M. Shaaban, "Beyond the bowel: Extraintestinal manifestations of inflammatory bowel disease," RadioGraphics, vol. 37, no. 4, pp. 1135-1160, 2017.

[6] R. Perez-Alamino, H. Maldonado-Ficco, and J. A. MaldonadoCocco, "Rheumatic manifestations in inflammatory bowel diseases: a link between GI and rheumatology," Clinical Rheumatology, vol. 35, no. 2, pp. 291-296, 2016.

[7] F. Atzeni, C. Defendenti, M. C. Ditto et al., "Rheumatic manifestations in inflammatory bowel disease," Autoimmunity Reviews, vol. 13, no. 1, pp. 20-23, 2014.

[8] M. C. Karreman, J. J. Luime, J. M. Hazes, and A. E. Weel, "The Prevalence and Incidence of Axial and Peripheral Spondyloarthritis in Inflammatory Bowel Disease: A Systematic Review and Meta-analysis," Journal of Crohn's and Colitis, vol. 11, pp. 631-642, 2017.
[9] C. Stolwijk, M. Pierik, R. Landewé, A. Masclee, and A. Van Tubergen, "Prevalence of self-reported spondyloarthritis features in a cohort of patients with inflammatory bowel disease," Canadian Journal of Gastroenterology \& Hepatology, vol. 27, no. 4, pp. 199-205, 2013.

[10] M. Rudwaleit, D. Van Der Heijde, R. Landewé et al., “The development of Assessment of SpondyloArthritis international Society classification criteria for axial spondyloarthritis (part II): validation and final selection," Annals of the Rheumatic Diseases, vol. 68, no. 6, pp. 777-783, 2009.

[11] R. Queiro, O. Maiz, J. Intxausti et al., "Subclinical sacroiliitis in inflammatory bowel disease: A clinical and follow-up study," Clinical Rheumatology, vol. 19, no. 6, pp. 445-449, 2000.

[12] I. Olivieri, F. Cantini, F. Castiglione et al., "Italian Expert Panel on the management of patients with coexisting spondyloarthritis and inflammatory bowel disease," Autoimmunity Reviews, vol. 13, no. 8, pp. 822-830, 2014.

[13] P. Conigliaro, M. S. Chimenti, M. Ascolani et al., "Impact of a multidisciplinary approach in enteropathic spondyloarthritis patients," Autoimmunity Reviews, vol. 15, no. 2, pp. 184-190, 2016.

[14] E. Naredo, I. Möller, E. de Miguel et al., "High prevalence of ultrasonographic synovitis and enthesopathy in patients with psoriasis without psoriatic arthritis: a prospective case-control study," Rheumatology, vol. 50, no. 10, pp. 1838-1848, 2011.

[15] R. Queiro, S. Alonso, M. Alperi et al., "Entheseal ultrasound abnormalities in patients with SAPHO syndrome," Clinical Rheumatology, vol. 31, no. 6, pp. 913-919, 2012.

[16] E. Naredo, R. J. Wakefield, A. Iagnocco et al., "The OMERACT ultrasound task force-status and perspectives," The Journal of Rheumatology, vol. 38, pp. 2063-2067, 2011.

[17] F. Bandinelli, M. Milla, S. Genise et al., "Ultrasound discloses entheseal involvement in inactive and low active inflammatory bowel disease without clinical signs and symptoms of spondyloarthropathy," Rheumatology, vol. 50, no. 7, pp. 1275-1279, 2011.

[18] J. Rovisco, C. Duarte, A. Batticcioto et al., "Hidden musculoskeletal involvement in inflammatory bowel disease: A multicenter ultrasound study," BMC Musculoskeletal Disorders, vol. 17, no. 1, article no. 84, 2016.

[19] A. Zabotti, F. Bandinelli, A. Batticciotto, C. A. Scirè, A. Iagnocco, and G. Sakellariou, "Musculoskeletal ultrasonography for psoriatic arthritis and psoriasis patients: A systematic literature review," Rheumatology, vol. 56, no. 9, pp. 1518-1532, 2017.

[20] M. Rudwaleit, R. Landewé, and D. van der Heijde, "The development of Assessment of SpondyloArthritis international Society classification criteria for axial spondyloarthritis (part I): classification of paper patients by expert opinion including uncertainty appraisal," Annals of the Rheumatic Diseases, vol. 68, pp. 770776, 2009. 


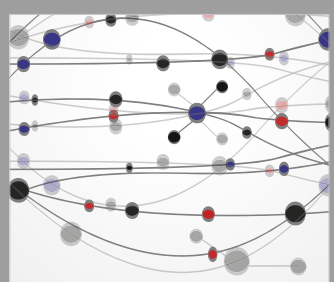

The Scientific World Journal
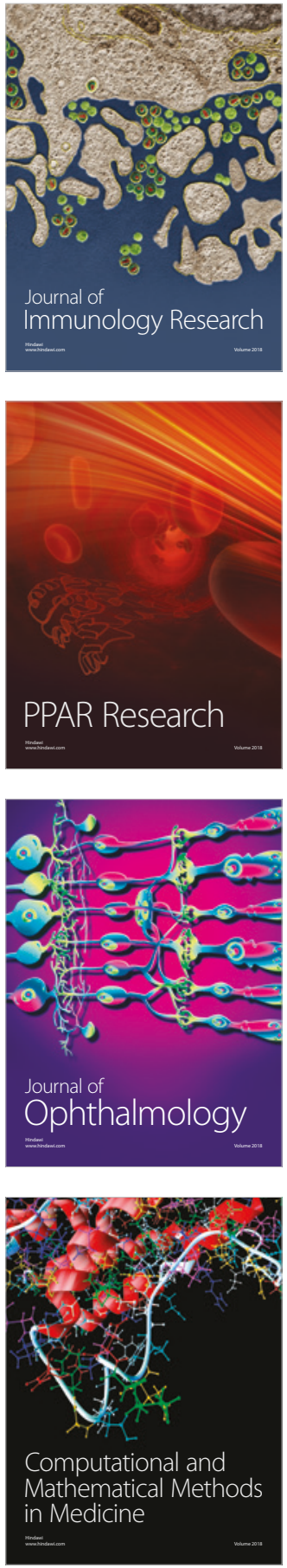

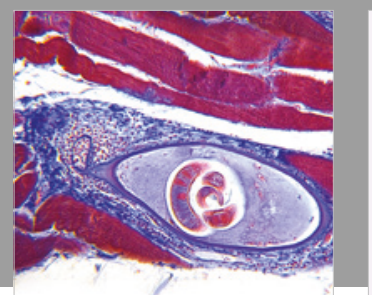

Gastroenterology Research and Practice

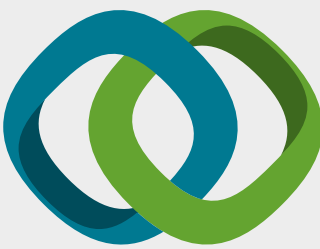

\section{Hindawi}

Submit your manuscripts at

www.hindawi.com
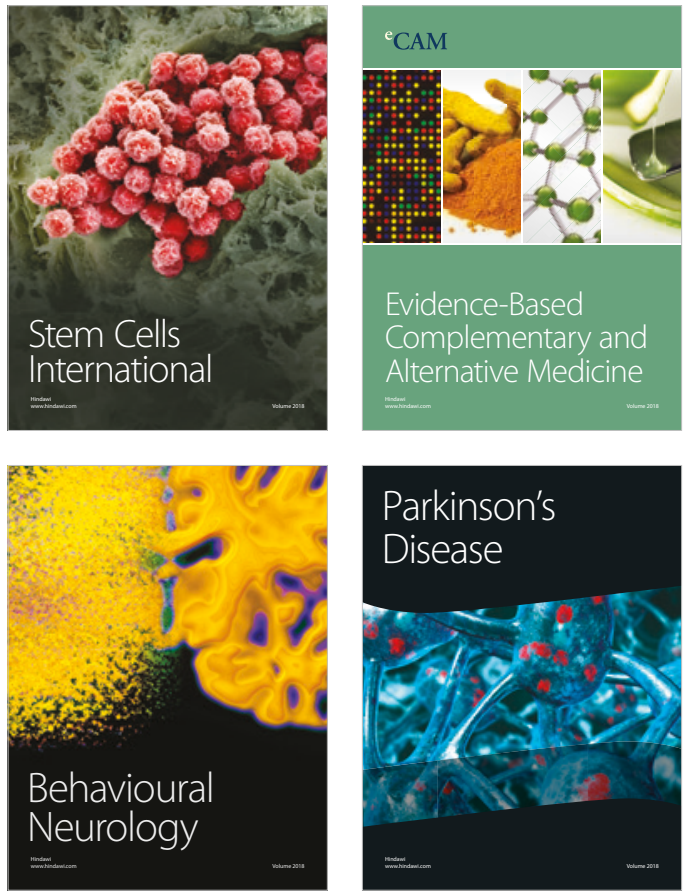

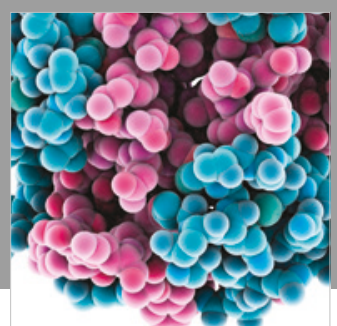

ournal of

Diabetes Research

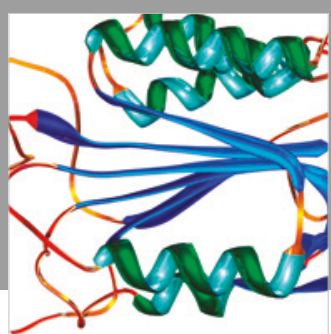

Disease Markers
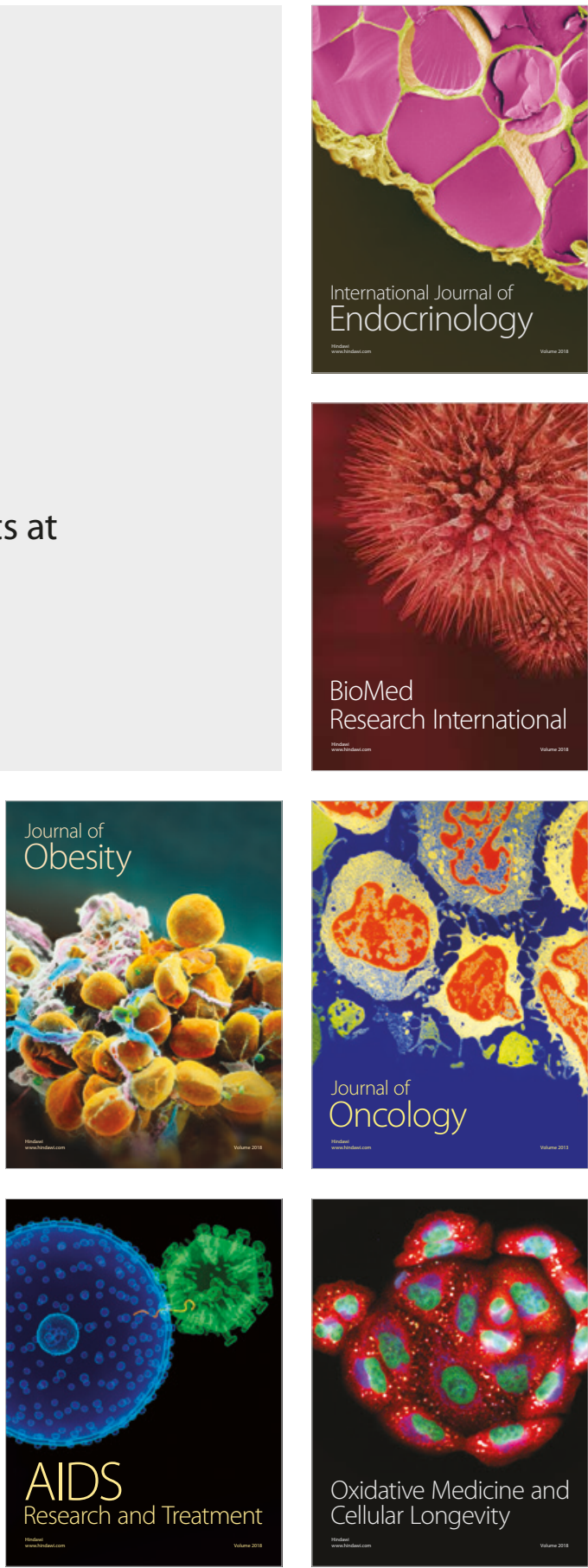